Review

\title{
Novel Molecular and Nanosensors for In Vivo Sensing
}

\author{
Mark A. Eckert ${ }^{1 *}$, Priscilla Q. Vu ${ }^{2}$, Kaixiang Zhang1,3, Dongku Kang2 ${ }^{2}$ M. Monsur Ali2 ${ }^{2}$ Chenjie $\mathrm{Xu}^{4}$ and \\ Weian Zhao ${ }^{1 凶}$
}

1. Department of Pharmaceutical Sciences, Department of Biomedical Engineering, Sue and Bill Gross Stem Cell Research Center and Chao Family Comprehensive Cancer Center, University of California, Irvine. 845 Health Sciences Road, Irvine, CA 92697, USA.

2. School of Medicine, University of California, Irvine, Irvine, CA 92697

3. Department of Chemistry, Beijing Key Laboratory for Analytical Methods and Instrumentation, Tsinghua University, Beijing, China 100084

4. Division of Bioengineering, School of Chemical and Biomedical Engineering, Nanyang Technological University, Singapore 637457, Singapore

* MAE and PQV contributed equally to this work.

\begin{abstract}
$\square$ Corresponding author: Weian Zhao. Email: weianz@uci.edu; Webpage: http://faculty.sites.uci.edu/zhaolab/
( ) Ivyspring International Publisher. This is an open-access article distributed under the terms of the Creative Commons License (http://creativecommons.org/ licenses/by-nc-nd/3.0/). Reproduction is permitted for personal, noncommercial use, provided that the article is in whole, unmodified, and properly cited.
\end{abstract}

Received: 2013.04.29; Accepted: 2013.06.14; Published: 2013.07.23

\begin{abstract}
In vivo sensors are an emerging field with the potential to revolutionize our understanding of basic biology and our treatment of disease. In this review, we highlight recent advances in the fields of in vivo electrochemical, optical, and magnetic resonance biosensors with a focus on recent developments that have been validated in rodent models or human subjects. In addition, we discuss major challenges in the development and translation of in vivo biosensors and present potential solutions to these problems. The field of nanotechnology, in particular, has recently been instrumental in driving the field of in vivo sensors forward. We conclude with a discussion of emerging paradigms and techniques for the development of future biosensors.
\end{abstract}

Key words: biosensors, molecular probes, in vivo imaging, in vivo sensing, nanoparticles, diagnostics

\section{Introduction}

At the most fundamental level, medicine is about understanding and correcting aberrations in normal biological function. To fully understand the dynamic and regulated processes underlying both normal biology and disease requires robust, sensitive and specific sensors of the molecular events underlying biology and pathology. Biosensors have been instrumental in building a foundation for our understanding of biology and have facilitated the rapid identification of novel drugs in screening assays [1]. The enormous amount of knowledge gained from in vitro biosensors has been a strong impetus for the translation of in vivo sensors. The development of in vivo biosensors that detect hypoxic conditions in tumors [2], sense caspase activity in response to therapeutics [3], and interrogate neuronal signaling in vivo [4] have confirmed in vitro observations and allowed functional assays not possible in vitro.

The need to develop in vivo sensors to directly interrogate biological processes in living organisms is driven by the fact that in vitro sensing often fails to fully capture the complexity of intact organ systems and are not able to continuously monitor biological events in situ. In addition, some biological events such as those that occur in the brain cannot realistically be measured in vitro. Informed by both biology and novel technologies, emerging in vivo sensors have begun to be applied in living systems to dynamically and continuously monitor biological processes. These technologies have the potential to accelerate detection 
of diseases such as cancer, inform treatments, and understand response to therapies.

Normally, a biosensor consists of a biorecognition element, a signal transducer, and a detector $[5,6]$. The recognition element, including antibodies, peptides, nucleic acids, or enzymes, is the portion of the sensor that initially binds to or interacts with the analyte or pathway of interest. In many cases this is associated with a conformational change, substrate cleavage, or enzymatic reaction that transduces the biorecognition event into a signal that may be detected via several modalities. For the purposes of this review, we will focus on three of the most well-characterized in vivo sensors: electrochemical, optical, and magnetic resonance (MR) sensors. Importantly we will also focus on sensors which interrogate dynamic processes to detect a biological activity, rather than imaging or staining probes for identification of cells or exogenous substances in vivo. As the fields of electrochemical [7-9], optical [6; 10; 11], and MR [12; 13] sensors are enormous and previously reviewed, we focus only on application of sensors to in vivo sensing to highlight the potential and challenges inherent to specific and sensitive sensing in living tissues. Although fluorescent proteins and genetic modification of organisms have greatly expanded our understanding of normal biology and disease processes, they have been extensively reviewed previously $[14 ; 15]$ and therefore are outside the scope of this review.

The application of biosensors to intact living systems has been hindered by both the inaccessibility of in vivo tissues and fundamental difficulties in sensor design and application. Sensing in vivo requires sensitive instruments capable of monitoring signals within a living system, while detectors must be biocompatible, nontoxic, and not perturb the system being examined. In recent years, however, an array of novel technologies have been developed and improved to overcome these challenges and allow monitoring of in vivo biology for the first time. This has involved both the development of novel techniques to improve biocompatibility and fundamental advances in detection technology.

From implantable devices for detecting cardiac damage [16] to in vivo sensors of cancer cell apoptosis [3], in vivo sensing has allowed unprecedented insight into multiple disease states. The enormous potential of in vivo sensing is perhaps best illustrated by the clinical approval of continuous in vivo glucose monitors for patients with diabetes. These devices have been clinically validated to improve glycemic control in patients by continuously monitoring glucose in the interstitial fluid of patients $[10,11]$. We anticipate that in the future a growing list of such devices and technologies will revolutionize healthcare. We hope that this review will serve to both highlight the breadth and potential of in vivo sensing as well as identify major unresolved challenges in translating in vivo sensors from the bench to the bedside.

\section{Electrochemical Sensors}

Electrochemical sensors are a well-established class of in vivo sensors which can offer near real-time measurement of analytes with implanted microelectrodes [19]. In general, electrochemical sensors function by taking advantage of the amperometric change associated with biological events, particularly those associated with enzymatic activity. For example, some glucose sensors utilize glucose oxidase electrochemically linked to an electrode to detect the electron transfer process associated with catalysis (Figure 1A). Electrochemical sensors typically require a sensor embedded within the tissue of interest that may be directly linked to signal processing units and power supplies via implanted wires, although wireless technologies are becoming more common. Spatial resolution is limited by the size and location of placement of the sensor itself. Since their development in 1973 [20], electrochemical sensors has been widely studied and improved over the last decades and have been used for detecting a range of in vivo targets such as glucose, glutamate, reactive nitrogen species and many neurochemicals [21;22].

From a practical point-of-view, glucose is the most widely employed target for in vivo electrochemical sensing which can be used for diabetes care and management [23]. The enormous incidence of diabetes coupled with the clinical utility of glucose sensing was the impetus for much of the work that has led to the development of clinically-approved in vivo glucose sensors. In most of these devices, glucose oxidase is immobilized on an electrode with biocompatible materials covered with a selectively permeable membrane such as Nafion or Polypyrrole to reduce signal interference. During the reaction:

$$
\text { Glucose }+\mathrm{O}_{2} \stackrel{\text { glucose oxidase }}{\longrightarrow} \text { gluconic acid }+\mathrm{H}_{2} \mathrm{O}_{2}
$$

Glucose concentration can be assayed by measuring the consumption of $\mathrm{O}_{2}$ or the production of $\mathrm{H}_{2} \mathrm{O}_{2}$ via electrochemical reduction or oxidation that occurs at the surface of a working electrode (Figure 1A). The major issues of implanted glucose sensors are the foreign body response, protease activity and instability. To address these problems, Wang et al synthesized dihydroxypropyl methacrylate (DHPMA) hydrogels with higher freezable water content, swelling rate, and uniform porosity that 
maximized the functional life of the sensor by minimizing fibrosis and inflammation [24]. Alternatively, glucose sensors have been embedded into poly(lactic-co-glycolic) acid (PLGA)/poly(vinyl alcohol) (PVA) composite hydrogels doped with the anti-inflammatory dexamethasone [25] (Figure 1B-D). The sensor-hydrogel composite continuously elutes the drug, leading to minimal foreign body response without affecting the sensitivity of the sensor. Interestingly, Park et al avoided the problems of enzyme instability by devising a nonenzymatic electrochemical sensor that makes use of nanoporous Pt electrodes for long term stability and sensitivity in glucose sensing [26]. Nanoporous Pt relatively selectively cat- alyzes the electrochemical oxidation of glucose; by incorporating an additional multilayered encapsulating polymer membrane, both selectivity and sensitivity were improved. Recently, a significant amount of attention has been directed at developing insulin sensors to complement glucose sensing. Although not yet tested in vivo, Gerasimov et al describe the generation of an insulin sensor in which an insulin-targeting aptamer was modified with a methylene blue redox probe; insulin concentration could be sensitively assayed with a detection limit of 10 to $50 \mathrm{nM}$ [27]. Similar approaches have clear utility in the incorporation of aptamers into electrochemical sensors.
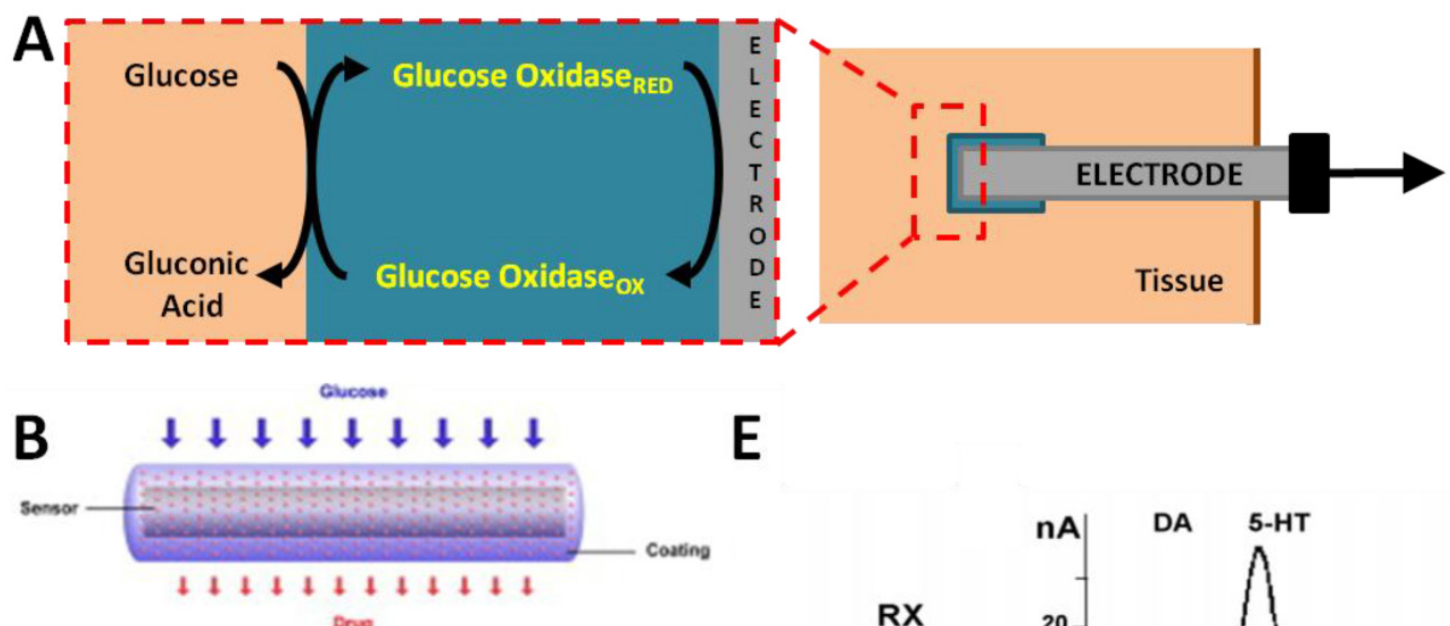

E
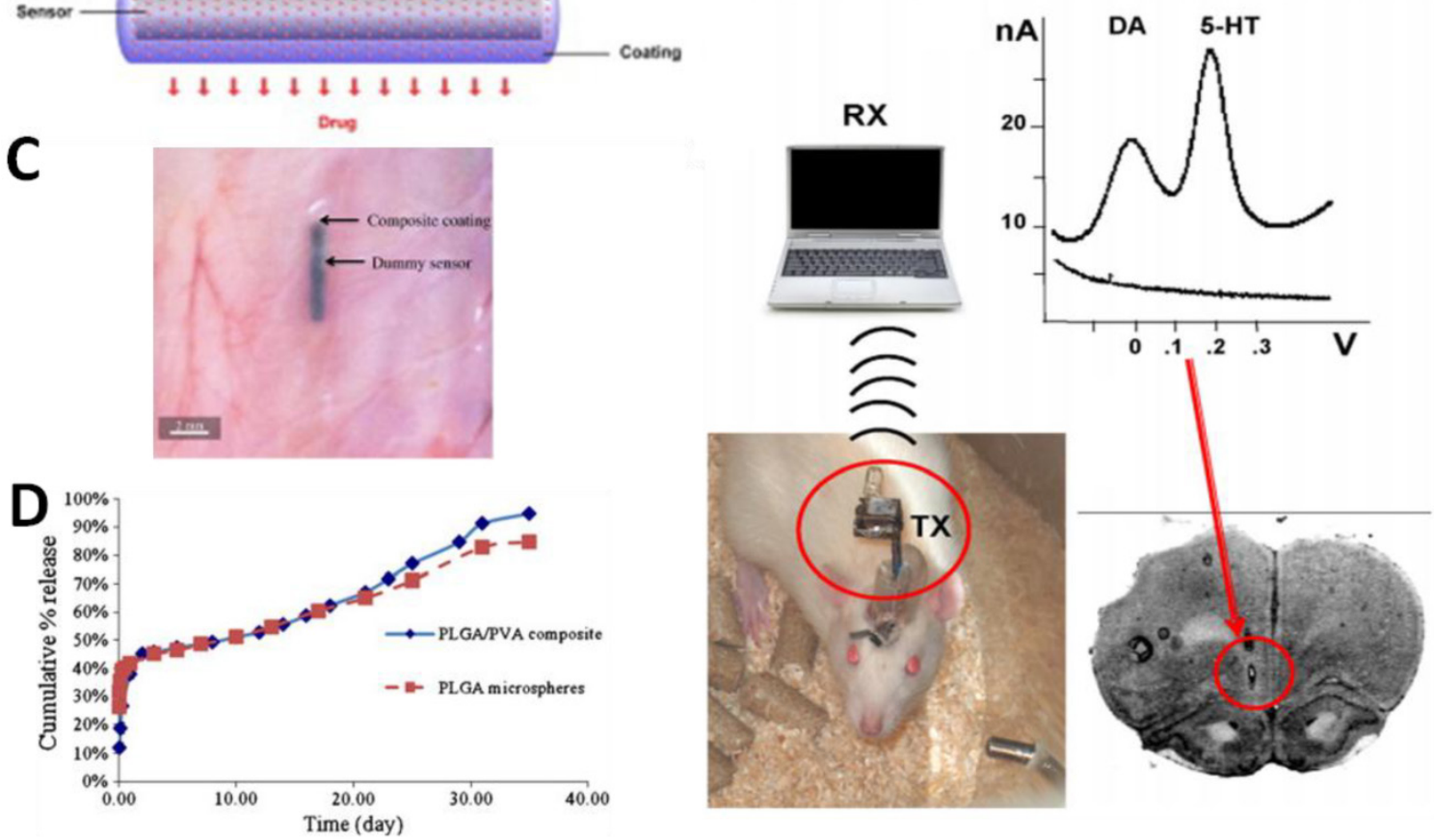

Figure I. Examples of in vivo electrochemical sensors. (A) Schematic of a typical electrochemical glucose sensor implanted in a tissue. Glucose oxidase is embedded into a matrix surrounding an electrode. In the presence of glucose, the glucose oxidase catalyzes its oxidation to gluconic acid. The electrode senses the electron transfer reaction of the glucose oxidase. (B) A "smart" glucose sensor developed by Wang et al can be encapsulated in an anti-inflammatory (dexamethasone) drug-eluting hydrogel to minimize foreign body response. (C) The "smart" sensor in situ demonstrating small size amenable to implantation. (D) The "smart" sensor has similar response to glucose concentration as an unmodified sensor while minimizing inflammatory responses [25]. (E) Implantable wireless electrochemical sensors of dopamine (DA) and serotonin (5-HT) may be embedded in the brain of rats to dynamically assay neurotransmitter activity in real time in freely-moving animals, as demonstrated by Crespi. An infrared transmitter (TX) transfers data to receiving station (RX) interfaced with a laptop for analysis. [29]. Panels B-D adapted from Wang et al 2013 with permission; panel E adapted from Crespi 2010 with permission. 
Recently, more prominent applications of in vivo electrochemistry have been in the field of neuroscience. As neurotransmitter signaling in the brain cannot be precisely studied with in vitro assays, the development of reliable electrochemical in vivo biosensors of neurotransmitters has revolutionized our understanding of the brain. In vivo monitoring of neurochemicals provides essential information on which networks are active and to what extent in the intact brain. Because many neurotransmitters are electrically active, generation of electrochemical sensors is relatively straightforward. For example, Phillips, et al measured sub-second dopamine release with carbon-fibre microelectrodes positioned into the nucleus accumbens of rats by fast-scan cyclic voltammetry (FSCV) [28]. FSCV is an electrochemical technique that allows measurement of the release and uptake dynamics of endogenous monoamine levels. It is mainly used to detect three major monoamine neurotransmitters, dopamine (DA), serotonin (5-HT), and norepinephrine (NE), as these substances can be oxidized at low voltages. Use of this technique allowed real-time measurements of dopamine release and uptake in the rodent brain in response to drugs and drug seeking behavior [28]. By incorporating wireless data transmission with implantable electrochemical sensors, real-time DA and 5-HT levels have been assayed in freely moving animals [29] (Figure 1E). Assessment of these pathways in response to dynamic environmental stimuli would clearly be impossible without the advent of implantable electrochemical biosensors. Similar studies have used in vivo electrochemical biosensors to examine events ranging from the kinetics of nitric oxide (NO) signaling in the brain following chemical stimulation [30] to the reaction of serotonin signaling to cytoskeletal disrupting agents [31]. The translation of such sensors to humans may be facilitated by recent advances in wireless information and power transfer to injectable biosensors. Power can be delivered to implanted sensors via radiofrequency (RF) generators or cutaneous induction coils [32; 33]; advances in wireless telemetry have produced RF and infrared wireless transmitters small enough to be implanted relatively non-invasively [22, 25].

Electrochemical biosensors have benefited greatly from advances in nanomaterials (e.g., gold nanoparticles, carbon nanotubes and graphene). As enzymes or antibodies bound to nanomaterials largely retain their bioactivity, nanomaterials may act as a scaffold for a large number of recognition elements [34]. In addition, nanomaterials can facilitate the electron transfer between enzyme and electrode or can themselves be used as electrodes. The use of gold nanoparticles in a glucose biosensor led to an over seven fold increase in electron transfer rate with decreased interference from oxygen [35]. In addition, graphene has recently shown utility in enhancing the detection of analytes, with a graphene-platinum nanoparticle hybrid electrosensor detecting cholesterol at levels as low as $0.2 \mu \mathrm{M}$ [36]. Nanoparticles may also be used to spatially organize electrochemical detectors. A needle-implantable electrochemical glucose sensor with excellent in vivo sensitivity was recently facilitated by generating a nanoporous working electrode via decoration with platinum nanoparticles [37]. As the diversity of nanomaterials increases, so does their potential utility in the next generation of electrochemical biosensors.

\section{Optical Sensors}

Optical sensors are based on generating or specifically localizing a visible or infrared light signal when bound by a target or acted on by a biological pathway. Fluorescence sensors may be realized by Förster resonance emission transfer (FRET), in which binding to an analyte brings two fluorophores together (or apart) to increase (or decrease) FRET fluorescence. Alternatively, the sensor may similarly employ a fluorophore-quencher pair in which the fluorescence increases dramatically upon separation of the pair. This has been realized, for example, by peptide sensors in which a fluorophore and quencher are separated by a sequence that is the substrate for a protease; upon being cleaved by the target protease, the fluorophore and quencher are separated and fluorescence increased (Figure 2A). Alternatively, a DNA-based sensor may undergo a conformational change upon binding to an analyte of interest that simultaneously separates the fluorophore and quencher to effect fluorescence (Figure 2B). In most cases, the fluorescence signal is detected with sensitive charge-coupled device cameras, although the development of implantable fiber optic detection systems has great potential for clinical application to glucose sensing in the future [38]. Fluorescence is a particularly appealing modality as it is relatively inexpensive with strong multiplexing capability due to the wide range of fluorophores available. Optical sensors have been designed to interrogate a variety of processes in vivo, from apoptosis to protease activity to hypoxia.

The most prominent examples of in vivo optical sensors have perhaps been in the field of cancer biology. Proteinase activity, particularly that of the matrix metalloproteinases (MMPs), is a hallmark of invasive cancers [39]. Optical sensors of proteinase activity take advantage of the fact that proteinases preferen- 
tially cleave specific peptide sequences. By separating a fluorophore and a quencher by a peptide linker that includes a proteinase cleavage site, local proteinase activity can be visualized as an increase in fluorescence. The first such sensors were cathepsin D sensors that incorporated fluorescence probes to allow detection of cathepsin D activity in vivo [32,33]. Subsequent sensors using MMP-specific cleavage peptides have revealed great specificity for invasive tumors and the capability of sensing MMP activity in deep-tissue tumors (Figure 2C-D) [42]. Spatial resolution of these techniques is sufficient to identify the location of the proteinase activity and the tumor with whole animal imaging. These sensors could have future utility in performing non-invasive optical "biopsies" to determine the invasiveness of tumors.

Sensor stability has also been utilized to generate an in vivo sensor of hypoxia. Low oxygen tension is

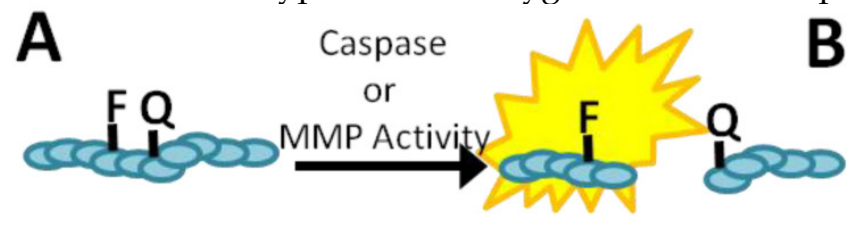

often associated with invasiveness in many cancers by stabilizing proteins such hypoxia inducible factor (HIF) with oxygen-dependent degradation (ODD) domains [43]. These proteins are rapidly degraded in the presence of oxygen, but stabilized by hypoxic conditions. By constructing a synthetic fluorescent peptide that includes an ODD domain, Kuchimaru et al visualized in vivo hypoxia by detecting the relative amount of fluorescence following administration of the recombinant sensor; signal could only be strongly generated when the peptide sensor was stabilized under hypoxic conditions [2]. This novel study confirmed in vitro evidence that the central areas of tumors are hypoxic in vivo, with increasing areas of hypoxia during tumor progression. Due to the ability to multiplex optical sensors, in the future it may be possible to sense multiple cellular events such as hypoxia and proteinase activity simultaneously.
B

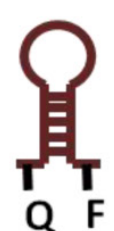

$\mathbf{F}$
Binding to

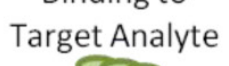

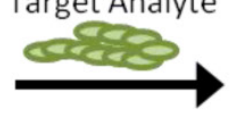
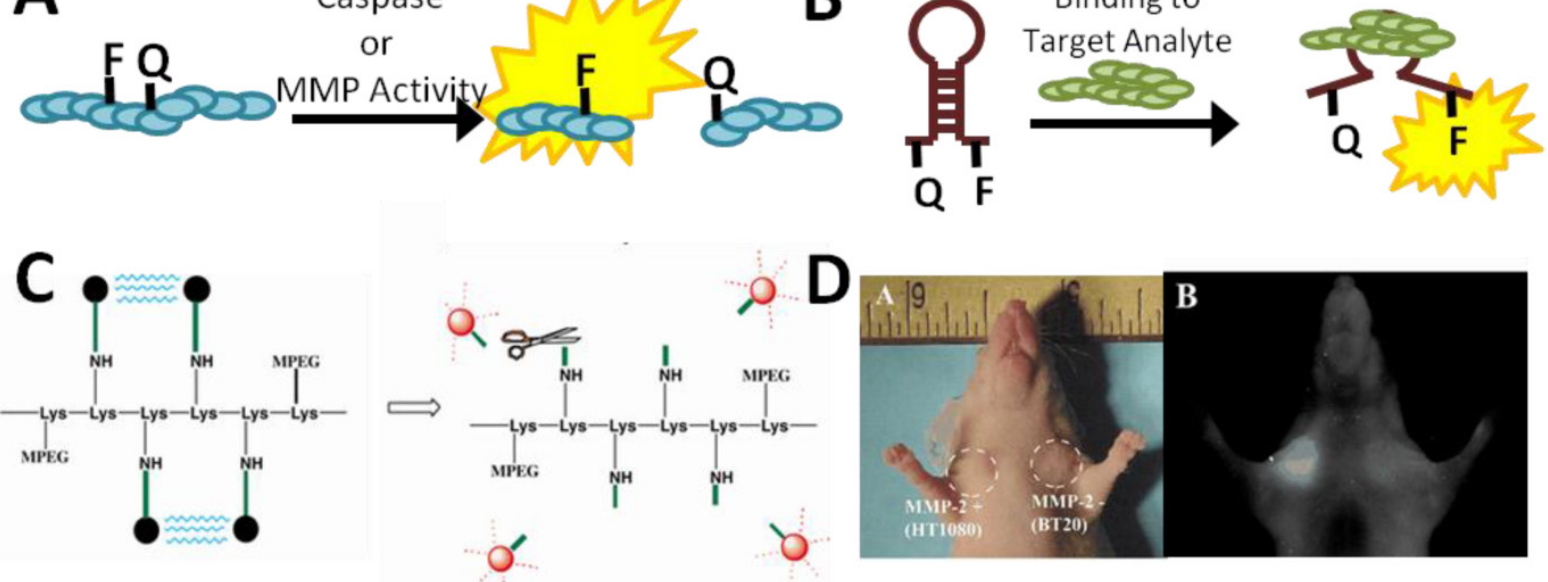

B

E

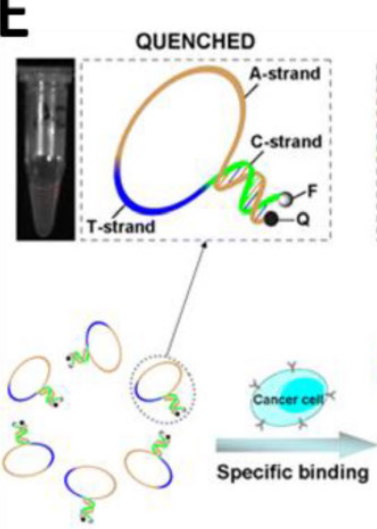

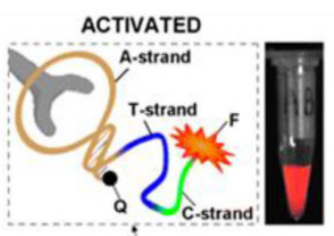

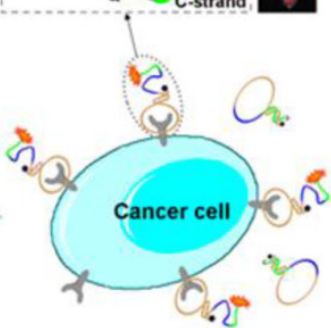

$\mathbf{F}$

Mouse without tumor implanted

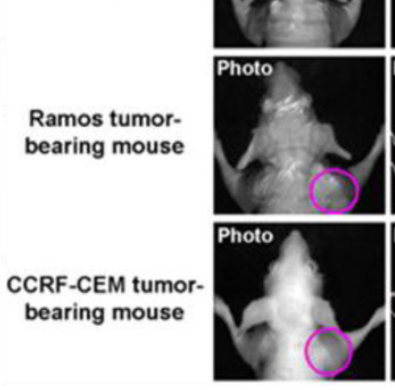

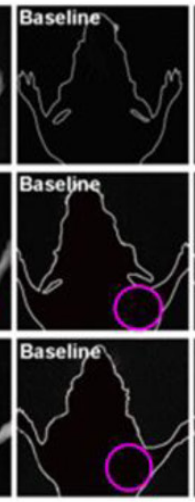

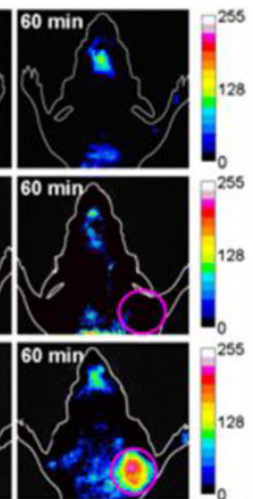

Figure 2. Examples of in vivo optical sensors. (A) Sensing may be achieved by the separation of a fluorophore-quencher pair on a sensor by the proteolytic activity of a metalloproteinase or caspase. (B) Switch-based sensors change conformation upon binding to an analyte of interest, leading to fluorescence due to separation of the fluorophore and quencher. (B) Schematic of a peptide-based MMP sensor. Fluorescence is quenched until cleavage of the peptide linking the fluorophores to a lysine backbone (Lys) by MMPs. (C) Cleavage of the MMP reporter in vivo can be assessed with whole animal imaging, which demonstrates a dramatic increase in fluorescence associated with HTI080 xenotransplants which express MMP2 compared to BT20 xenografts, which do not [42]. (D) A switch-based, aptamer biosensor for CCRF-CEM cancer cells undergoes a conformational change upon binding to its target cells which leads to a dramatic increase in fluorescence. (E) In vivo fluorescence is clearly observed in CCRF-CEM tumor-bearing mice, but not control mice [45]. Panels C-D adapted from Bremer et al $200 \mathrm{I}$ with permission; panels E-F adapted from Shi et al 20I I with permission. 
Understanding the response of a cancer to therapy is currently difficult but important in dictating subsequent therapeutic interventions. Therefore a simple, non-invasive means of measuring apoptosis in response to therapy would be a tremendous asset for clinicians. Stefflova et al recently designed just such a system, in which a photodynamic therapeutic was coupled with a fluorescent molecular reporter for caspase 3 activity [3]. The reporter consisted of a fluorophore separated from a quencher by a caspase 3 cleavable sequence (KGDEVDGSGK). In the presence of apoptotic cells, in which caspase 3 is activated, the probe is cleaved, potentiating an increase in near-infrared fluorescence. In addition, soluble probes referred to as fluorescent labeled inhibitors of caspases (FLICA) have been developed for imaging caspase activity [44]. These peptides mimic endogenous caspase substrates with the addition of a fluoromethyl ketone group facilitating irreversible and specific binding to activated caspases. FLICA probes have been successfully used in vivo to identify apoptotic cells in tumors following chemotherapy via intravital microscopy. Similar probes for other cellular activities would clearly have clinical utility in cancer and other diseases.

Switch-based DNA sensors, in which fluorescence is produced or quenched in the presence of an analyte or biological activity, have also found utility in cancer biology. Switch-based sensors have particular utility in vivo as the reversibility of the switch-based system allows dynamic responses to continuously variable signals [6]. The utility of this approach was recently validated in a publication by Shi et al in which they designed a switch-based fluorescent activatable aptamer probe (AAP) for detection of leukemia cell lines in vivo [45] (Figure 2E-F). The AAP was based on a previously-identified aptamer capable of binding acute lymphoblastic leukemia (CCRF-CEM) cells but not other cancer or normal cells. When bound to target CCRF-CEM cells, the APP increased in fluorescence, allowing sensitive and specific detection of small numbers of leukemia cells in vivo. The switch-based nature of the sensor had major advantages in selectivity and specificity compared to always-on reporters which allowed for fine spatial resolution of tumors with whole animal imaging. This paper provides valuable proof-of-concept of the feasibility of in vivo switch-based and aptamer-based sensors.

The relative simplicity of optical sensors, particularly switch-based sensors, makes them amenable to modifications that include tethering them to the surface of cells. We recently demonstrated that a switch-based DNA sensor for platelet-derived growth factor (PDGF), a ligand important in multiple disease processes including cancer, could be tethered to the surface of adult stem cells without affecting cell viability or migration to the bone marrow [46]. By harnessing the tropism of various cells for specific organs or disease states, it will be possible to specifically deliver reporters to spatial areas of interest. This may prove to have utility in both reducing background signal in off-target organs, as well as improving sensitivity in target sites. In the future, both prokaryotic and eukaryotic cells may have advantages in the delivery of sensors in vivo.

\section{Magnetic Resonance Sensors}

Magnetic resonance (MR) based sensors are intriguing platforms for in vivo sensing due to their high spatial resolution and capability of interrogating deep tissues in intact specimens. Although optical sensors have had great utility in mouse models, deep tissues in mice are still much shallower than similar organs in humans, potentially precluding the use of even NIR probes in humans. Most MR-based biosensors utilize magnetic nanoparticles to interrogate the function of an organ or system. These nanoparticles function as contrast agents that alter the T1 or T2 relaxation time of nearby protons; by incorporating biosensing elements on these particles, sensing functionality can be incorporated into the particles. Sensing may be achieved by binding of magnetic particles to an analyte of interest to induce a local magnetic signal, or by being acting on by biological molecules to unveil a magnetic signal. For example, cleavage of a peptide linker on the surface of the MR sensor by proteases may lead to increased retention of the sensor at tumor sites (Figure 3A). Alternatively, bifunctional nanoparticles may bind to a single analyte, leading to altered $\mathrm{T} 1$ or $\mathrm{T} 2$ relaxation times that may be quantified in the presence of an analyte (Figure 3B). Although a major limitation of MR-based sensors is relatively poor temporal resolution due to the amount of time required for signal collection, they have still found wide utility, especially in sensing cellular activities in deep tissues.

Harris et al recently described an in vivo MR sensor for proteinase activity associated with cancers [47] (Figure 3A \& C). In this system, a nanoparticle was coated with protease-cleavable ligands that masked a cell internalization signal embedded in the ligand. In the presence of MMPs, however, the ligand was cleaved to expose the internalization signal. At this point, the nanoparticle may be internalized by cells. In the presence of a tumor, the particles accumulated inside the tumor due to high local expression of proteases. Similar reporter systems utilizing other 
proteases, or a combination of protease-specific linkers, could lead to efficient reporters for multiple cancer types as well as other pathologies that include disregulation of MMP activity. The use of MR as a sensing modality rather than optical sensing has clear advantages in sensing of protease activity in the deep tissues characteristic of many tumors.

Early detection of apoptosis following heart attacks can be critical in optimizing care and minimizing further cardiac damage. Dash et al recently demonstrated the feasibility of detecting cardiac apoptosis using MR sensing of superparamagnetic iron oxide (SPIO) nanoparticles conjugated with recombinant annexin- $\mathrm{V}$ [48]. During apoptosis, phosphadylserine is externalized to the outside of the cell membrane, allowing binding of annexin- $\mathrm{V}$. The apoptosis sensor was sensitive and able to spatially resolve apoptosis in a rodent model of nonischemic cardiac damage. Although initially validated in a cardiac injury model, this system has clear potential utility in assessing apoptosis in other disease and treatment models, such as following chemotherapy for cancers.
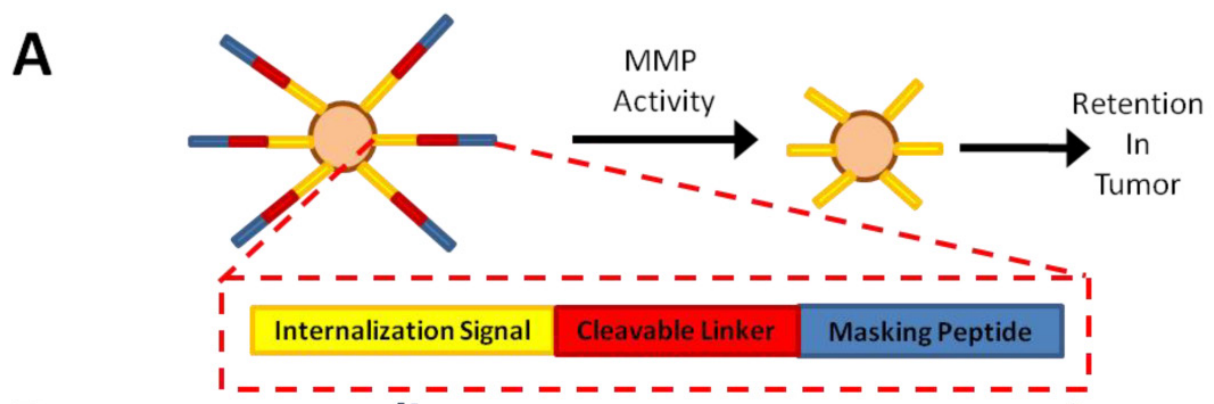

B
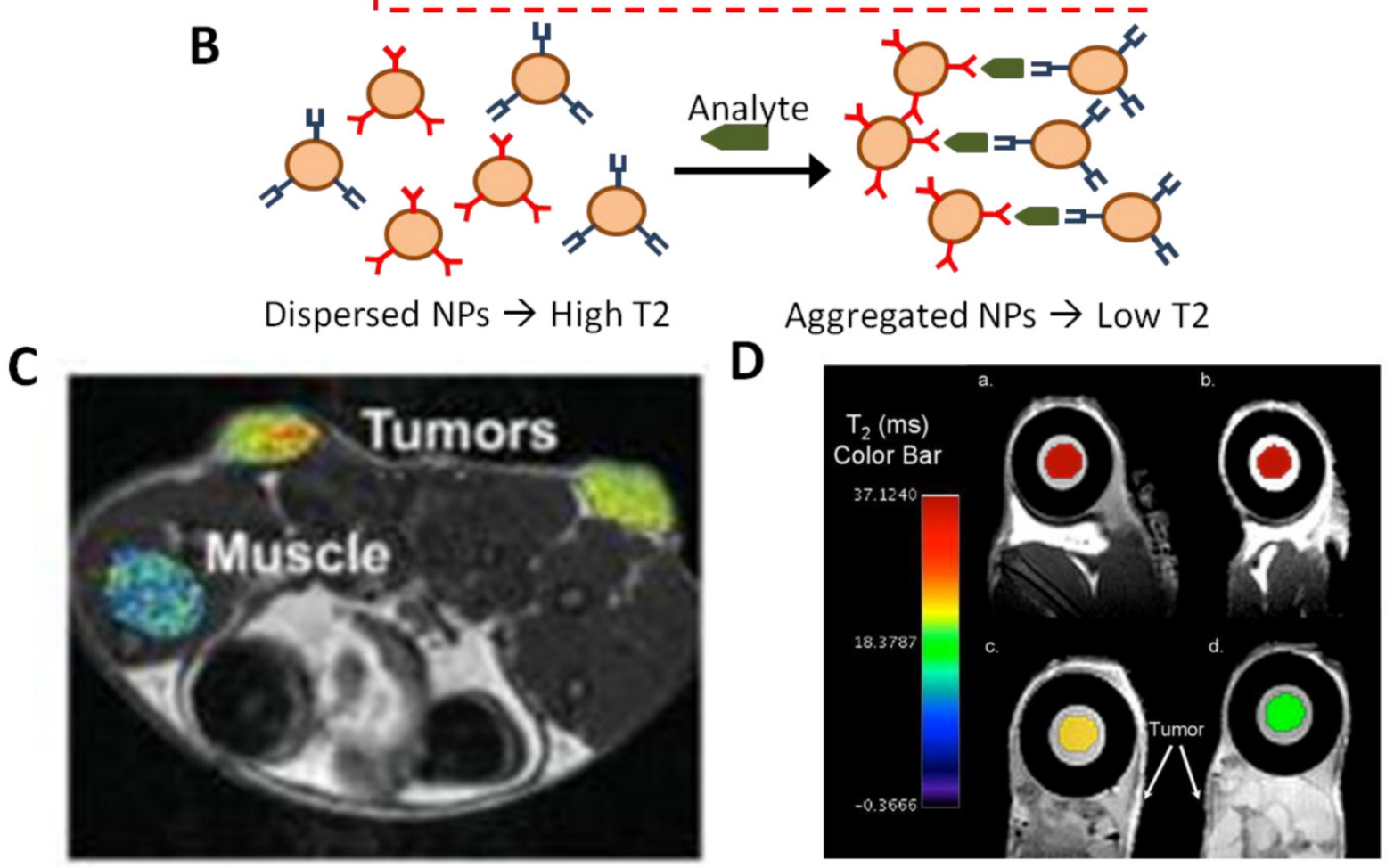

Figure 3. Examples of in vivo MR sensors. (A) Masking of magnetic nanoparticles by a protease-cleavable ligand prevents internalization until the mask is removed by tumor-associated MMP activity. The nanoparticles can then be efficiently internalized by the adjacent tumor cells. (B) Alternatively, dispersed, bifunctional nanoparticles have a high T2 MR signal; when bound to their target analyte, they aggregate, quantitatively lowering the T2 signal. (C) Tumor-specific magnetic contrast can easily be visualized in areas of MMP2 activity associated with tumors [47]. (D) Implantable MR sensors of cancer biomarkers are incorporated into a polycarbonante membrane. In the presence of biomarkers, the MR signal is dynamically quenched [50]. Panel $\mathrm{C}$ adapted from Harris et al 2008 with permission; panel D adapted from Daniel et al 2009 with permission. 
As stem-cell based regenerative medicine becomes more feasible, there is increasing interest in tracking the viability of transplanted cells, with MR-based sensors being a particularly appealing modality for non-invasively sensing the viability of cells in deep tissues. Recently, Chan et al applied MR sensing to assess transplanted cell viability in vivo by creating a $\mathrm{pH}$-sensitive magnetic nanosensor [49]. In this approach, $\mathrm{pH}$-sensitive sensors were encapsulated in hydrogels co-injected with transplanted hepatocytes; acidification associated with cell death was specifically and dynamically imaged via both optical and MR-based methods. This approach is particularly appealing, as it was accomplished with all clinical-grade reagents implying that it may be translated clinically relatively quickly.

MR sensors have also recently been incorporated into implantable sensor devices. A device has been engineered as an implantable diagnostic device for monitoring the cancer biomarker, human chorionic gonadotrophin (hCG-beta), in-vivo [50] (Figure 3B \& D). The device uses a semi-permeable membrane that contains nanoparticle magnetic relaxation switches $(\mathrm{MRSw})$, which are magnetic nanoparticles with a super paramagnetic iron oxide core and a cross-linked dextran shell. MRSw accumulate on a desired analyte, changing the relaxitivity in that region; hCG-beta concentration can be easily quantified via MR imaging in vivo with this technique. A similar technique was used to create an implantable sensor for multiple biomarkers associated with myocardial infarction [47]. The system was sensitive and capable of dynamically interrogating changes in biomarker concentration following infarction in a rodent model. As a platform technology, implantable sensors could potentially revolutionize both diagnosis and monitoring of progression and response in multiple diseases.

Many of the advances in MR sensing modalities has been possible due to the development of novel superparamagnetic iron nanoparticles with interesting geometries and physical properties that improve sensitivity, specificity, and biocompatibility [51]. With sizes of less than $20 \mathrm{~nm}$, when exposed to an external magnetic field they will align with the field leading to robust and specific magnetic saturation [51]. Magnetic nanoparticles are easily modified with functional groups such as antibodies or peptide sensors via simple click chemistry techniques [52]. These novel particles promise to expand the feasibility of using MR sensing to assay a wider range of analytes in the future. Improvements in MR detector technology and design may also improve access to what is now a relatively expensive imaging modality.

\section{Overcoming the Challenges of In Vivo Sensing}

The ideal in vivo biosensor would be non-toxic to the host, biocompatible, stable over long periods of time, and sensitive with appropriate temporal and spatial resolution for the system being interrogated. The capability of multiplexing sensors would be a clear advantage of any sensor. Any sensor must accurately interrogate the system in question with minimal perturbation of normal biology. A variety of techniques and approaches have been adopted in the field to address the special challenges of in vivo sensing (Table 1).

Table I: Challenges in the Development of In Vivo Sensors

\begin{tabular}{|c|c|}
\hline Challenge & Example Solutions \\
\hline Toxicity & $\begin{array}{l}\text { Optimization of physical parameters of nanoparticles [73] } \\
\text { Biocompatible coatings to manipulate biodistribution and clearance of nanoparticles [54] }\end{array}$ \\
\hline Biocompatibility & $\begin{array}{l}\text { Biomimetic and "smart" hydrogels to minimize foreign body response [25] } \\
\text { Micellar, biocompatible coatings [54] }\end{array}$ \\
\hline Sensitivity & $\begin{array}{l}\text { Enhanced binding properties via bio-inspired multivalency [62] } \\
\text { Signal amplification via "nanoparticle communication" to take advantage of endogenous signal am- } \\
\text { plification cascades [63] }\end{array}$ \\
\hline Resolution & $\begin{array}{l}\text { Improved intravital microscopy, including three-photon microscopy [66] } \\
\text { Miniaturization of electrochemical detection elements [33] }\end{array}$ \\
\hline Targeted Delivery of Sensors In Vivo & $\begin{array}{l}\text { Targeted delivery of biosensors via cell-surface engineering [46] } \\
\text { Localized internalization via masking with protease-cleavable ligands [47] }\end{array}$ \\
\hline Invasive Implantation of Sensors & $\begin{array}{l}\text { Development of wireless power supplies and signal transducers [33] } \\
\text { Improved miniaturization technology via nanopatterning [37] }\end{array}$ \\
\hline Multiplexing & $\begin{array}{l}\text { Use of multiple sensing modalities (optical + MR, electrochemical + optical, etc) } \\
\text { SERS has ideal characteristics for multiplex sensing [67] } \\
\text { Development of novel sensing modalities to expand sensing palette [72] }\end{array}$ \\
\hline
\end{tabular}


Toxicity has been an enduring concern with the in vivo use of nanoparticles. Due to their size and reactivity with biological molecules including proteins, there is potential for immune reaction or coagulation [53]. In addition, clearance by the reticuloendothelial and renal system can both limit the bioavailability of the sensors as well as potentially induce damage to the host [54]. Optimization of the physical parameters of the sensor can maximize the in vivo half-life of the sensor, while surface coatings can be introduced to minimize toxicity or bioreactivity. Alternatively, lipid-based encapsulations, such as micellar encapsulation with phospholipids, have improved the in-vivo biocompatibility of quantum dots for sensing applications [55]. Importantly, amphiphilic polymer coated quantum dots conjugated to streptavidin are now commercially available [56].

Embedded biosensors, such as electrochemical glucose sensors, avoid the problems of clearance, but must still minimize immunogenicity and scar tissue formation around the implantation site [57]. The recent development of polymeric "smart" coatings may minimize the foreign body response via local elution of anti-inflammatories [25]. In addition, advances in miniaturization and wireless power and data transmission promise to reduce the invasiveness of many electrochemical in vivo sensors [33].

The most outstanding issues facing optical sensors involve reliable and accurate detection of signals in vivo. Tissues and biological fluids are relatively opaque to visible light, limiting the depth to which signals may be non-invasively monitored. In addition, autofluorescence from tissues and the environment compound the difficulty of sensitive and specific sensing [58]. Advances in the generation of molecular probes with wavelengths in the infrared range, often facilitated by nanotechnology, will doubtlessly extend the range and application of optical sensors [59-61]. Improvements in sensor design, sensitivity, and miniaturization also have clear roles in expanding the ease with which optical sensors may be applied to clinical sensors.

A major challenge inherent in the use of nanoparticle-based sensors is signal amplification. Ghosh et al recently used the bacteriophage M13 as a scaffold to display targeting ligands and numerous nanoparticles for the detection of cancer cells in mice. The multivalent binding of the bacteriphage scaffold coupled with the capability of targeting a larger number of nanoparticles to the tumor lead to significantly improved sensitivity and selectivity [62]. Furthermore, the Bhatia lab recently developed a novel solution to this problem by engineering nanoparticles that can "communicate" in vivo to amplify tumor targeting [63]. In this elegant system, nanoparticles target tumor sites where they then locally activate the coagulation cascade to broadcast tumor location to clot-targeting nanoparticles. This project lays the foundation for novel future technologies that may take advantage of endogenous signaling events to solve the problem of signal amplification. In addition, cell surface engineering offers an appealing method of locally delivering sensors to in vivo compartments of interest to minimize background and increase selectivity of the sensor [46]. These methods have the effect of greatly increasing the sensitivity of the nanosensors while preserving their selectivity for the tumor.

Apart from sensor properties and design, improvements in detector technology have the potential to greatly expand the utility of both current and future in vivo sensors. Currently, many in vivo sensors utilize confocal microscopy, a time consuming and expensive technique. Advances in the development of embedded optical sensors have clear potential utility in the detection of analytes in which spatial resolution is not a priority $[56,57]$. On the other hand, however, cutting edge microscopy techniques are also expanding the palette of optical sensors. For example, three-photon confocal microscopy, now possible with ZnS nanocrystals and other nano-scale materials, has exquisite spatial resolution, minimal background, and excellent tissue penetrance. Most recently, this technique has been applied to imaging of tumors, but could easily be expanded or modified for other sensing purposes [66].

\section{Future Directions}

Much of the future of in vivo sensing depends on addressing the challenges discussed above. Solving the problems of robust detection, stability, biocompatibility and multiplexing will allow us to unlock the true potential of in vivo sensors in understanding and treating human disease. We anticipate that nanoparticles, innovative signal amplification techniques, and alternative sensing modalities will have particularly large impacts in the future of in vivo sensing.

The field of nanotechnology, including nanoparticles, nanocages, rods, shells, and graphene-based particles, is an emerging field with enormous potential to contribute to in vivo sensing. Nanoparticles can act as scaffolds for immobilization of detection elements, enhance electron transfer, catalyze electrochemical reactions, or act as reactant themselves. Surface modification of nanoparticles can dramatically improve biocompatibility, half life, and biodistribution. As discussed above, nanoparticles are ca- 
pable of profoundly enhancing the electrical properties of electrochemical sensing elements. Recently, in vivo multiplex detection of multiple cancer biomarkers in vivo was made possible via the use of antibody-decorated NIR nanoparticles to generate nanotags for surface-enhanced Raman scattering (SERS) of epidermal growth factor receptor (EGFR) and human epidermal growth factor 2 (Her 2) [67]. Up to three-fold multiplexing was achieved, with excellent sensitivity and specificity. The widespread adoption of nanoparticle-based sensors will depend in large part on characterizing their safety, both acute and long-term.

In addition to the electrochemical, optical, and MR-based sensing modalities discussed above, several novel sensing technologies have been explored in vivo. In addition to SERS, a wide range of positron emission tomography (PET) sensors exist, particularly for metabolism (18F-fludeoxyglucose, FDG-PET [68]) and neutrotransmitters (11C-metomidate for sensing enzyme activities of adrenocortical tumors [69] and $11 \mathrm{C}-\mathrm{McN} 5652$ for sensing serotonin uptake in the brain [70]). As mentioned above, SERS has great potential, particularly in multiplex detection with up to ten different SERS nanotags being resolvable in vivo [71]. Photoacoustic sensors, which measure electromagnetic energy via acoustic detection promises to aid in multiplexing and deep tissue sensing due to the excellent tissue penetrance of acoustic energy and minimal acoustic background in vivo [72]. The selection of sensing modality or combination of modalities in the future will depend on the nature of the process being interrogated.

The future of in vivo sensing is bright, with advances in detection technology coinciding with the development of an array of useful sensors and techniques to improve biocompatibility and safety. Major advances in improving the safety and reliability of in vivo sensors has culminated in the clinical approval of continuous glucose monitors for diabetic patients with associated improvements in glucose control and health. Optimization and translation of sensors for everything from cancer to cardiac arrest will require a multidisciplinary effort between physicians, scientists, and engineers.

\section{Abbreviations}

5-HT: serotonin; AAP: activatable aptamer probe; DA: dopamine; DHPMA: dihydroxypropyl methacrylate; EGFR: epidermal growth factor receptor; FDG: fludeoxyglucose; FLICA: fluorescent labeled inhibitors of caspases; FRET: Förster resonance emission transfer; FSCV: fast-scan cyclic voltammetry; hCG: human chorionic gonadotrophin; Her 2: human epidermal growth factor 2; HIF: hypoxia inducible factor; MMP: matrix metalloproteinase; MR: magnetic resonance; MRSw: magnetic relaxation switches; NE: norepinephrine; NO: nitric oxide; ODD: oxygen-dependent degradation; PDGF: platelet-derived growth factor; PET: positron emission tomography; PLGA: poly(lactic-co-glycolic) acid; PVA: poly(vinyl alcohol); RF: radiofrequency; SERS: surface-enhanced Raman scattering; SPIO: superparamagnetic iron oxide

\section{Acknowledgment}

This work is supported by the start-up fund from the Department of Pharmaceutical Sciences, Sue and Bill Gross Stem Cell Research Center and the Chao Family Comprehensive Cancer Center at UC Irvine, and NCI Cancer Center Support Grant 5P30CA062203-18. M.A.E is supported by a California Institute for Regenerative Medicine (CIRM) Training Grant (TG2-01152). Panels 1B-D adapted from Wang Y, Papadimitrakopoulos F, Burgess DJ. Polymeric "smart" coatings to prevent foreign body response to implantable biosensors. Journal of Controlled Release 2013; 169 (3): 341-7 with permission. Panel 1E adapted from Crespi F. Wireless in vivo voltammetric measurements of neurotransmitters in freely behaving rats. Biosensors E Bioelectronics 2010; 25 (11): 2425-30 with permission. Panels 2C-D adapted from Bremer C, Bredow S, Mahmood U, Weissleder R, Tung CH. Optical imaging of matrix metalloproteinase-2 activity in tumors: feasibility study in a mouse model. Radiology 2001; 221 (2): 523-9 with permission. Panels 2E-F adapted from Shi H, He X, Wang K, Wu X, Ye X, Guo Q, Tan W, Qing Z, Yang X, Zhou B. Activatable aptamer probe for contrast-enhanced in vivo cancer imaging based on cell membrane protein-triggered conformation alteration. Proceedings of the National Academy of Sciences of the United States of America 2011; 108 (10) 3900-5 with permission. Panel 3C adapted from Harris TJ, Von Maltzahn G, Lord ME, Park J-H, Agrawal A, Min D-H, Sailor MJ, Bhatia SN. Small 2008; 4 (9): 1307-12 with permission. Panel 3D adapted from Daniel KD, Kim GY, Vassiliou CC, Galindo M, Guimaraes AR, Weissleder R, Charest A, Langer R, Cima MJ. Biosensors \& Bioelectronics 2009; 24 (11): 3252-7 with permission.

\section{Competing Interests}

The authors have declared that no competing interest exists.

\section{References}

1. Cooper MA. Optical biosensors in drug discovery. Nature reviews. Drug discovery 2002; 1 (7): 515-28. 
2. Kuchimaru T, Kadonosono T, Tanaka S, Ushiki T, Hiraoka M, Kizaka-Kondoh S. In vivo imaging of HIF-active tumors by an oxygen-dependent degradation protein probe with an interchangeable labeling system. PloS one 2010; 5 (12): e15736.

3. Stefflova K, Chen J, Li H, Zheng G. Targeted photodynamic therapy agent with a built-in apoptosis sensor for in vivo near-infrared imaging of tumor apoptosis triggered by its photosensitization in situ. Molecular imaging 2006;5 (4): 520-32.

4. Lama RD, Charlson K, Anantharam A, Hashemi P. Ultrafast detection and quantification of brain signaling molecules with carbon fiber microelectrodes. Analytical chemistry 2012; 84 (19): 8096-101.

5. Wilson GS, Gifford R. Biosensors for real-time in vivo measurements. Biosensors \& bioelectronics 2005; 20 (12): 2388-403.

6. Plaxco KW, Soh HT. Switch-based biosensors: a new approach towards real-time, in vivo molecular detection. Trends in biotechnology 2011; 29 (1): $1-5$.

7. Wang J. Electrochemical glucose biosensors. Chemical reviews 2008; 108 (2): 814-25.

8. Ronkainen NJ, Halsall HB, Heineman WR. Electrochemical biosensors. Chemical Society reviews 2010; 39 (5): 1747-63.

9. Vaddiraju S, Tomazos I, Burgess DJ, Jain FC, Papadimitrakopoulos F. Emerging synergy between nanotechnology and implantable biosensors: a review. Biosensors $\mathcal{E}$ bioelectronics 2010; 25 (7): 1553-65.

10. Dai N, Kool ET. Fluorescent DNA-based enzyme sensors. Chemical Society reviews 2011; 40 (12): 5756-70.

11. Vallée-Bélisle A, Plaxco KW. Structure-switching biosensors: inspired by Nature. Current opinion in structural biology 2010; 20 (4): 518-26.

12. James ML, Gambhir SS. A molecular imaging primer: modalities, imaging agents, and applications. Physiological reviews 2012; 92 (2): 897-965.

13. Haun JB, Yoon T-J, Lee H, Weissleder R. Magnetic nanoparticle biosensors. Wiley interdisciplinary reviews. Nanomedicine and nanobiotechnology 2010;2 (3): 291-304.

14. Giepmans BNG, Adams SR, Ellisman MH, Tsien RY. The fluorescent toolbox for assessing protein location and function. Science (New York, N.Y.) 2006; 312 (5771): 217-24.

15. Rao J, Dragulescu-Andrasi A, Yao H. Fluorescence imaging in vivo: recent advances. Current opinion in biotechnology 2007; 18 (1): 17-25.

16. Ling $\mathrm{Y}$, Pong $\mathrm{T}$, Vassiliou CC, Huang PL, Cima MJ. Implantable magnetic relaxation sensors measure cumulative exposure to cardiac biomarkers. Nature biotechnology 2011; 29 (3): 273-7.

17. Gerritsen M, Jansen JA, Lutterman JA. Performance of subcutaneously implanted glucose sensors for continuous monitoring. The Netherlands journal of medicine 1999; 54 (4): 167-79.

18. Raccah D, Sulmont V, Reznik Y, Guerci B, Renard E, Hanaire H, et al. Incremental value of continuous glucose monitoring when starting pump therapy in patients with poorly controlled type 1 diabetes: the RealTrend study. Diabetes care 2009; 32 (12): 2245-50.

19. Wilson GS, Johnson MA. In-vivo electrochemistry: what can we learn about living systems? Chemical reviews 2008; 108 (7): 2462-81.

20. Kissinger PT, Hart JB, Adams RN. Voltammetry in brain tissue--a new neurophysiological measurement. Brain research 1973; 55 (1): 209-13.

21. Wassum KM, Tolosa VM, Wang J, Walker E, Monbouquette HG, Maidment NT. Silicon Wafer-Based Platinum Microelectrode Array Biosensor for Near Real-Time Measurement of Glutamate in Vivo. Sensors (Basel, Switzerland) 2008; 8 (8): 5023-5036.

22. Griveau S, Dumézy C, Séguin J, Chabot GG, Scherman D, Bedioui F. In vivo electrochemical detection of nitric oxide in tumor-bearing mice. Analytical chemistry 2007; 79 (3): 1030-3.

23. Pickup J. Developing glucose sensors for in vivo use. Trends in biotechnology 1993; 11 (7): 285-91.

24. Wang C, Yu B, Knudsen B, Harmon J, Moussy F, Moussy Y. Synthesis and performance of novel hydrogels coatings for implantable glucose sensors. Biomacromolecules 2008; 9 (2): 561-7.

25. Wang Y, Papadimitrakopoulos F, Burgess DJ. Polymeric "smart" coatings to prevent foreign body response to implantable biosensors. Journal of controlled release : official journal of the Controlled Release Society 2013; 169 (3): 341-7.

26. Park S, Park S, Jeong R-A, Boo H, Park J, Kim HC, et al. Nonenzymatic continuous glucose monitoring in human whole blood using electrified nanoporous Pt. Biosensors \& bioelectronics 2012; 31 (1): 284-91.

27. Gerasimov JY, Schaefer CS, Yang W, Grout RL, Lai RY. Development of an electrochemical insulin sensor based on the insulin-linked polymorphicregion. Biosensors \& bioelectronics 2013; 42: 62-8.

28. Phillips PEM, Stuber GD, Heien MLA V, Wightman RM, Carelli RM. Subsecond dopamine release promotes cocaine seeking. Nature 2003; 422 (6932): 614-8.
29. Crespi F. Wireless in vivo voltammetric measurements of neurotransmitters in freely behaving rats. Biosensors \& bioelectronics 2010; 25 (11): 2425-30.

30. Barbosa RM, Lourenço CF, Santos RM, Pomerleau F, Huettl P, Gerhardt GA, et al. In vivo real-time measurement of nitric oxide in anesthetized rat brain. Methods in enzymology 2008; 441: 351-67.

31. Crespi F. Further Electrochemical and Behavioural Evidence of a Direct Relationship Between Central 5-HT and Cytoskeleton in the Control of Mood. The open neurology journal 2010; 4: 5-14.

32. Chang C-W, Chiou J-C. A Wireless and Batteryless Microsystem with Implantable Grid Electrode/3-Dimensional Probe Array for ECoG and Extracellular Neural Recording in Rats. Sensors (Basel, Switzerland) 2013; 13 (4): 4624-39.

33. Kim T -i., McCall JG, Jung YH, Huang X, Siuda ER, Li Y, et al. Injectable, Cellular-Scale Optoelectronics with Applications for Wireless Optogenetics. Science 2013; 340 (6129): 211-216.

34. Luo X, Morrin A, Killard AJ, Smyth MR. Application of Nanoparticles in Electrochemical Sensors and Biosensors. Electroanalysis 2006; 18 (4): 319-326.

35. Xiao Y, Patolsky F, Katz E, Hainfeld JF, Willner I. "Plugging into Enzymes": nanowiring of redox enzymes by a gold nanoparticle. Science (New York, N.Y.) 2003; 299 (5614): 1877-81.

36. Dey RS, Raj CR. Development of an Amperometric Cholesterol Biosensor Based on Graphene-Pt Nanoparticle Hybrid Material. The Journal of Physical Chemistry C 2010; 114 (49): 21427-21433.

37. Vaddiraju S, Legassey A, Qiang L, Wang Y, Burgess DJ, Papadimitrakopoulos F. Enhancing the Sensitivity of Needle-Implantable Electrochemical Glucose Sensors via Surface Rebuilding. Journal of diabetes science and technology 2013; 7 (2): 441-451.

38. Tierney S, Falch BMH, Hjelme DR, Stokke BT. Determination of glucose levels using a functionalized hydrogel-optical fiber biosensor: toward continuous monitoring of blood glucose in vivo. Analytical chemistry 2009; 81 (9): 3630-6.

39. Hanahan D, Weinberg RA. Hallmarks of cancer: the next generation. Cell 2011; 144 (5): 646-74.

40. Tung $\mathrm{CH}$, Bredow S, Mahmood U, Weissleder R. Preparation of a cathepsin D sensitive near-infrared fluorescence probe for imaging. Bioconjugate chemistry 1999;10 (5): 892-6.

41. Tung $\mathrm{CH}$, Mahmood U, Bredow S, Weissleder R. In vivo imaging of proteolytic enzyme activity using a novel molecular reporter. Cancer research 2000; 60 (17): 4953-8.

42. Bremer C, Bredow S, Mahmood U, Weissleder R, Tung CH. Optical imaging of matrix metalloproteinase-2 activity in tumors: feasibility study in a mouse model. Radiology 2001; 221 (2): 523-9.

43. Chan DA, Sutphin PD, Yen S-E, Giaccia AJ. Coordinate regulation of the oxygen-dependent degradation domains of hypoxia-inducible factor 1 alpha. Molecular and cellular biology 2005; 25 (15): 6415-26.

44. Lee BW, Olin MR, Johnson GL, Griffin RJ. In vitro and in vivo apoptosis detection using membrane permeant fluorescent-labeled inhibitors of caspases. Methods in molecular biology (Clifton, N.J.) 2008; 414: 109-35.

45. Shi H, He X, Wang K, Wu X, Ye X, Guo Q, et al. Activatable aptamer probe for contrast-enhanced in vivo cancer imaging based on cell membrane protein-triggered conformation alteration. Proc. Natl. Acad. Sci. U. S. A. 2011; 108 (10): 3900-5.

46. Zhao W, Schafer S, Choi J, Yamanaka YJ, Lombardi ML, Bose S, et al. Cell-surface sensors for real-time probing of cellular environments. Nature nanotechnology 2011; 6 (8): 524-31.

47. Harris TJ, Von Maltzahn G, Lord ME, Park J-H, Agrawal A, Min D-H, et al. Protease-triggered unveiling of bioactive nanoparticles. Small (Weinheim an der Bergstrasse, Germany) 2008; 4 (9): 1307-12.

48. Dash R, Chung J, Chan T, Yamada M, Barral J, Nishimura D, et al. A molecular MRI probe to detect treatment of cardiac apoptosis in vivo. Magnetic resonance in medicine: official journal of the Society of Magnetic Resonance in Medicine / Society of Magnetic Resonance in Medicine 2011; 66 (4): 1152-62.

49. Chan KWY, Liu G, Song X, Kim H, Yu T, Arifin DR, et al. MRI-detectable $\mathrm{pH}$ nanosensors incorporated into hydrogels for in vivo sensing of transplanted-cell viability. Nature materials 2013; 12 (3): 268-75.

50. Daniel KD, Kim GY, Vassiliou CC, Galindo M, Guimaraes AR, Weissleder R, et al. Implantable diagnostic device for cancer monitoring. Biosensors \& bioelectronics 2009; 24 (11): 3252-7.

51. $\mathrm{Xu} \mathrm{C}$, Sun S. New forms of superparamagnetic nanoparticles for biomedical applications. Advanced drug delivery reviews 2012; 65 (5): $732-43$.

52. McCarthy JR, Weissleder R. Multifunctional magnetic nanoparticles for targeted imaging and therapy. Advanced drug delivery reviews 2008; 60 (11): 1241-51. 
53. Love SA, Maurer-Jones MA, Thompson JW, Lin Y-S, Haynes CL. Assessing nanoparticle toxicity. Annual review of analytical chemistry (Palo Alto, Calif.) 2012; 5: 181-205.

54. Li S-D, Huang L. Nanoparticles evading the reticuloendothelial system: role of the supported bilayer. Biochimica et biophysica acta 2009; 1788 (10): 2259-66.

55. Dubertret B, Skourides P, Norris DJ, Noireaux V, Brivanlou AH, Libchaber A. In vivo imaging of quantum dots encapsulated in phospholipid micelles. Science (New York, N.Y.) 2002; 298 (5599): 1759-62.

56. Wu X, Liu H, Liu J, Haley KN, Treadway JA, Larson JP, et al. Immunofluorescent labeling of cancer marker Her2 and other cellular targets with semiconductor quantum dots. Nature biotechnology 2003; 21 (1): 41-6.

57. Kenneth Ward W. A review of the foreign-body response to subcutaneously-implanted devices: the role of macrophages and cytokines in biofouling and fibrosis. Journal of diabetes science and technology 2008; 2 (5): 768-77.

58. Mansfield JR, Gossage KW, Hoyt CC, Levenson RM. Autofluorescence removal, multiplexing, and automated analysis methods for in-vivo fluorescence imaging. Journal of biomedical optics 2005;10 (4): 41207.

59. Bentolila LA, Ebenstein $Y$, Weiss S. Quantum dots for in vivo small-animal imaging. Journal of nuclear medicine: official publication, Society of Nuclear Medicine 2009; 50 (4): 493-6.

60. Villa C, Erratico S, Razini P, Fiori F, Rustichelli F, Torrente Y, et al. Stem cell tracking by nanotechnologies. International journal of molecular sciences 2010; 11 (3): 1070-81.

61. Shen $\mathrm{H}$, Zhang L, Liu M, Zhang Z. Biomedical applications of graphene. Theranostics 2012; 2 (3): 283-94.

62. Ghosh D, Lee Y, Thomas S, Kohli AG, Yun DS, Belcher AM, et al. M13-templated magnetic nanoparticles for targeted in vivo imaging of prostate cancer. Nature nanotechnology 2012; 7 (10): 677-82.

63. Von Maltzahn G, Park J-H, Lin KY, Singh N, Schwöppe C, Mesters R, et al. Nanoparticles that communicate in vivo to amplify tumour targeting. Nature materials 2011; 10 (7): 545-52.

64. Ziegler KJ. Developing implantable optical biosensors. Trends in biotechnology 2005; 23 (9): 440-4.

65. O'Sullivan T, Munro EA, Parashurama N, Conca C, Gambhir SS, Harris JS, et al. Implantable semiconductor biosensor for continuous in vivo sensing of far-red fluorescent molecules. Optics express 2010; 18 (12): 12513-25.

66. Yu JH, Kwon S-H, Petrášek Z, Park OK, Jun SW, Shin K, et al. High-resolution three-photon biomedical imaging using doped $\mathrm{ZnS}$ nanocrystals. Nature materials 2013; 12 (4): 359-66.

67. Maiti KK, Dinish US, Samanta A, Vendrell M, Soh K-S, Park S-J, et al. Multiplex targeted in vivo cancer detection using sensitive near-infrared SERS nanotags. Nano Today 2012; 7 (2): 85-93.

68. Juweid ME, Cheson BD. Positron-emission tomography and assessment of cancer therapy. The New England journal of medicine 2006; 354 (5): 496-507.

69. Sundin A. Imaging of adrenal masses with emphasis on adrenocortical tumors. Theranostics 2012; 2 (5): 516-22.

70. Frankle WG, Huang Y, Hwang D-R, Talbot PS, Slifstein M, Van Heertum $\mathrm{R}$, et al. Comparative evaluation of serotonin transporter radioligands 11C-DASB and 11C-McN 5652 in healthy humans. Journal of nuclear medicine: official publication, Society of Nuclear Medicine 2004; 45 (4): 682-94.

71. Zavaleta CL, Smith BR, Walton I, Doering W, Davis G, Shojaei B, et al. Multiplexed imaging of surface enhanced Raman scattering nanotags in living mice using noninvasive Raman spectroscopy. Proc. Natl. Acad. Sci. U. S. A. 2009; 106 (32): 13511-6.

72. Kottmann J, Grob U, Rey JM, Sigrist MW. Mid-infrared fiber-coupled photoacoustic sensor for biomedical applications. Sensors (Basel, Switzerland) 2013; 13 (1): 535-49.

73. Kim T-H, Kim M, Park H-S, Shin US, Gong M-S, Kim H-W. Size-dependent cellular toxicity of silver nanoparticles. Journal of biomedical materials research. Part A 2012; 100 (4): 1033-43. 